\title{
Neurogenetic analysis of childhood disintegrative disorder
}

Abha R. Gupta ${ }^{1,2^{*}+}$, Alexander Westphal ${ }^{2,3 \dagger}$, Daniel Y. J. Yang ${ }^{2}$, Catherine A. W. Sullivan ${ }^{1}$, Jeffrey Eilbott ${ }^{2}$, Samir Zaidi', Avery Voos ${ }^{2}$, Brent C. Vander Wyk ${ }^{2}$, Pam Ventola², Zainulabedin Waqar' ${ }^{1}$, Thomas V. Fernandez ${ }^{2,3}$, A. Gulhan Ercan-Sencicek ${ }^{2}$, Michael F. Walker ${ }^{2}$, Murim Choi ${ }^{4}$, Allison Schneider ${ }^{2}$, Tammy Hedderly ${ }^{5}$, Gillian Baird ${ }^{5}$, Hannah Friedman ${ }^{2}$, Cara Cordeaux ${ }^{2}$, Alexandra Ristow ${ }^{2}$, Frederick Shic ${ }^{2}$,Fred R. Volkmar ${ }^{2}$ and Kevin A. Pelphrey ${ }^{2}$

\begin{abstract}
Background: Childhood disintegrative disorder (CDD) is a rare form of autism spectrum disorder (ASD) of unknown etiology. It is characterized by late-onset regression leading to significant intellectual disability (ID) and severe autism. Although there are phenotypic differences between CDD and other forms of ASD, it is unclear if there are neurobiological differences.

Methods: We pursued a multidisciplinary study of $\operatorname{CDD}(n=17)$ and three comparison groups: low-functioning ASD $(n=12)$, high-functioning ASD ( $n=50)$, and typically developing $(n=26)$ individuals. We performed whole-exome sequencing (WES), copy number variant (CNV), and gene expression analyses of CDD and, on subsets of each cohort, non-sedated functional magnetic resonance imaging (fMRI) while viewing socioemotional (faces) and non-socioemotional (houses) stimuli and eye tracking while viewing emotional faces.

Results: We observed potential differences between CDD and other forms of ASD. WES and CNV analyses identified one or more rare de novo, homozygous, and/or hemizygous (mother-to-son transmission on chrX) variants for most probands that were not shared by unaffected sibling controls. There were no clearly deleterious variants or highly recurrent candidate genes. Candidate genes that were found to be most conserved at variant position and most intolerant of variation, such as TRRAP, ZNF236, and KIAA2018, play a role or may be involved in transcription. Using the human BrainSpan transcriptome dataset, CDD candidate genes were found to be more highly expressed in non-neocortical regions than neocortical regions. This expression profile was similar to that of an independent cohort of ASD probands with regression. The non-neocortical regions overlapped with those identified by fMRI as abnormally hyperactive in response to viewing faces, such as the thalamus, cerebellum, caudate, and hippocampus. Eye-tracking analysis showed that, among individuals with ASD, subjects with CDD focused on eyes the most when shown pictures of faces.
\end{abstract}

Conclusions: Given that cohort sizes were limited by the rarity of CDD, and the challenges of conducting non-sedated fMRI and eye tracking in subjects with ASD and significant ID, this is an exploratory study designed to investigate the neurobiological features of CDD. In addition to reporting the first multimodal analysis of CDD, a combination of fMRI and eye-tracking analyses are being presented for the first time for low-functioning individuals with ASD. Our results suggest differences between CDD and other forms of ASD on the neurobiological as well as clinical level.

Keywords: Autism spectrum disorder (ASD), Childhood disintegrative disorder (CDD), Regression, Intellectual disability (ID), Genetics, Functional magnetic resonance imaging (fMRI), Eye tracking

\footnotetext{
*Correspondence: abha.gupta@yale.edu

${ }^{\dagger}$ Equal contributors

'Department of Pediatrics, Yale School of Medicine, New Haven, Connecticut, USA

${ }^{2}$ Child Study Center, Yale School of Medicine, New Haven, Connecticut, USA

Full list of author information is available at the end of the article
} 


\section{Background}

Autism spectrum disorder (ASD) is defined by deficits in social communication and interaction and restricted, repetitive patterns of behavior, interests, or activities [1]. Decades before Kanner published his landmark paper describing autism [2], Heller reported on six normally developing children who experienced a severe regression in skills between 3 and 4 years of age leading to global impairments, including autistic features [3, 4]. Heller termed the condition dementia infantilis, which was included in the ICD-10 [5] and DSM-IV [6] as childhood disintegrative disorder (CDD). CDD was defined by normal development for at least the first 2 years of life followed by regression before age 10 years in at least two of the following areas: (1) expressive or receptive language, (2) social skills or adaptive behavior, (3) bowel or bladder control, (4) play, and (5) motor skills. There has been much debate as to whether CDD is a late-onset variant of autism or a distinct entity $[7,8]$. CDD was subsumed by the diagnosis ASD in the DSM-5 [1], since there was little scientific basis for including CDD as a separate disorder [9].

There are, however, important phenotypic differences between CDD and other forms of ASD [8, 10-15]. While symptoms of ASD are usually recognized by 2 years of age, the onset of symptoms in CDD is usually between 3 and 4 years of age. While approximately a third of children with ASD experience a regression in skills, again usually by age 2 years [16], CDD is defined by regression, which is characteristically of later onset, more global in extent, and more severe in degree. Indeed, children with CDD generally have the poorest outcome among individuals with ASD, usually with severe loss of cognitive and communication skills $[8,11]$. In contrast to $\mathrm{CDD}$, children who are diagnosed with ASD later than the typical age range tend to be higher functioning, leading to the delay in diagnosis, and early subtle abnormalities are often noted in retrospect $[10,12]$. The majority of children with CDD experience a distinct prodrome characterized by bouts of anxiety and terror $[3,4$, $8,17]$. No consistent medical, environmental, or psychosocial triggers have been associated with CDD [8].

Our overarching question is whether there are neurobiological features that distinguish CDD from other forms of ASD. The genetic basis, neuroimaging abnormalities, and social phenotype of ASD are being intensively studied, but no similarly comprehensive studies have been published examining CDD for two important reasons. First, CDD is rare. While the prevalence of ASD is reported to be $1 / 68$ [18], the prevalence of CDD is estimated to be 1-2/100,000 [19]. Second, conducting experimental protocols such as non-sedated functional magnetic resonance imaging (fMRI) and eye tracking with low-functioning subjects is extremely challenging.
To study CDD, we used a multidisciplinary approach encompassing: (1) expert clinical characterization; (2) the identification of candidate genes and gene expression analysis; (3) an analysis of brain function, via fMRI, in response to viewing socioemotional (fearful faces) and non-socioemotional (houses) stimuli; and (4) the precise quantification of the social behavioral phenotype using eye tracking. This study is novel not only in examining the neurobiological features of CDD but also in obtaining a combination of reliable non-sedated fMRI and eyetracking data from low-functioning individuals on the autism spectrum.

\section{Methods}

A detailed description of all methods can be found in Additional file 1: Supplementary information. We studied four cohorts: (1) subjects with $\operatorname{CDD}(n=17$, Table 1$)$, (2) low-functioning [full-scale IQ $(F S I Q) \leq 75$ ] subjects with ASD (LFASD, $n=12$ ) and early-onset delays ( $<2$ years old), (3) high-functioning (FSIQ $\geq 75$ ) subjects with ASD (HFASD, $n=50$ ) and early-onset delays ( $<2$ years old), and (4) typically developing subjects (TD, $n=26)$. The genetics analysis focused on the CDD cohort whereas the fMRI and eye-tracking analyses included subsets of each cohort. We performed wholeexome sequencing (WES) and copy number variant (CNV) analyses of 15 families affected by CDD, which included 15 probands, 13 unaffected sibling controls, and their parents (Additional file 2: Table S1), to identify three types of rare [novel or found at most once across 1000 Genomes (May 2011 release), NHLBI GO ESP Exome Variant Server (ESP6500SI-V2), and inhouse database of 2500 exomes] protein-changing variants: (1) de novo, (2) homozygous, and (3) hemizygous (mother-to-son transmission on chrX). We included one additional category for family CDD17 since the father and paternal grandfather reportedly have highfunctioning autism: paternally inherited likely genedisrupting (LGD) variants (premature stop codon, splice site disruption, deletion). We used the human BrainSpan exon-array transcriptome dataset [20] to plot the brain expression profile of CDD candidate genes and conduct co-expression analysis. To study neural systems, we used non-sedated fMRI, and a blocked design involving the presentation of grayscale fearful face (NimStim set of facial expressions) [21] and house (lab database) images to determine brain activation patterns across the four cohorts [CDD $(n=7)$, LFASD $(n=7)$, HFASD $(n=14)$, and TD $(n=19)]$. To quantify the social phenotype of our four cohorts [CDD $(n=5)$, LFASD $(n=7)$, HFASD $(n=32)$, and TD $(n=14)]$, we collected eye-tracking data as they viewed emotional faces [21]. 


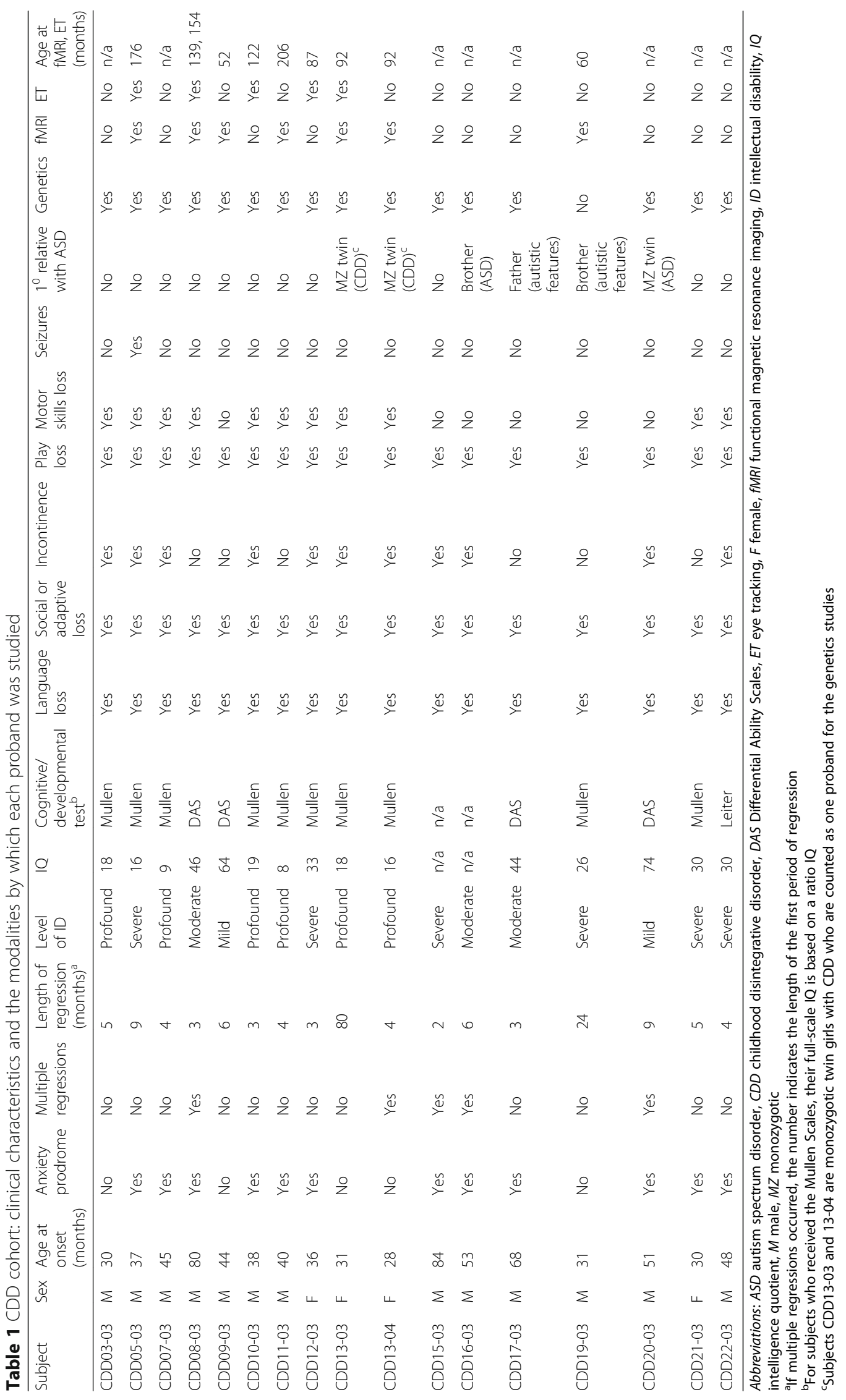




\section{Results}

\section{Study subjects}

Clinical characteristics of the CDD cohort and the number of subjects examined by each study modality are shown in Table 1. The sex ratio of 3.25 males to 1 female is similar to that reported for ASD. The mean and median age at onset of symptoms was 46 and 40 months, respectively, with a range of 28 to 84 months. Seventy percent of subjects experienced a prodrome of anxiety and terror. Thirty percent of subjects had multiple episodes of regression. The length of the first regressive episode ranged from 2 months to almost 7 years in one subject. Most subjects have severe to profound ID, with the mean and median IQ being 30 and 26, respectively, with a range of 8 to 74 . All had loss of language skills, loss of social skills or adaptive behavior, and loss of play skills. Sixty-five percent had loss of bowel or bladder control, and the same proportion had loss of motor skills. Although CDD has been reported to be almost always sporadic, a few of our subjects have immediate family members with ASD or autistic features, including two sets of monozygotic twins. Both members of one pair (CDD13-03/04) have CDD; in the other pair (CDD20-03/04), one has CDD and the other has ASD.

\section{Genetics}

Given the rarity, severity, and apparently sporadic transmission seen in most CDD cases, we hypothesized that rare variants of large effect contribute to the etiology. Indeed, there is abundant evidence for the contribution of rare variants to ASD [22-24]. As shown in Table 2, we found one or more rare variants for all but one proband, which were not shared by any unaffected sibling controls (Additional file 2: Table S2). We also looked for compound heterozygous variants in subjects by searching for additional variants in genes affected by de novo variants but did not find any. The rates of all high-probability (Bayesian quality score $\geq 50$ ) de novo variants were 0.80 / proband exome and 0.92/sibling exome (Additional file 2: Table S3), which are similar to the overall rates calculated from 11 recent WES studies of neurodevelopmental disorders: 1.00 /proband exome, $n=2358$; 0.82 /control exome, $n=731$ [25]. There were no significant differences in the rates of non-synonymous de novo, homozygous, and hemizygous variants (Additional file 2: Table S3); the rate of brain-expressed genes affected; phylogenetic $P$ value (PhyloP) conservation scores at variant positions; Residual Variation Intolerance Scores (RVIS); and polymorphism phenotyping v2 (PolyPhen-2) scores (Additional file 2: Table S2) between the probands and siblings.

We found one de novo genic CNV in a proband $(0.07 /$ proband, Table 2), which is similar to rates previously reported for ASD [26], and none in siblings. The proband
$\mathrm{CNV}$ is a $2 \mathrm{~kb}$ heterozygous deletion of the 3'UTR of $O G D H L$, which encodes a component of a mitochondrial protein complex implicated in neurodegeneration [27]. One gene, SUPT2OHL2, and two gene families, USP and $B B S$, are affected in more than one CDD proband. Two hemizygous missense variants were identified in SUPT20HL2, which encodes a putative transcription factor but could be a pseudogene according to the UniProtKB database (http://www.uniprot.org/). Three members of the USP (ubiquitin-specific peptidase) gene family are affected in CDD probands: USP9X (hemizygous missense), USP9Y (paternally-inherited non-sense), and USP26 (hemizygous missense). They encode deubiquitinating enzymes that prevent the degradation of proteins. Two members of the Bardet-Biedel Syndrome (BBS) gene family, which is involved in ciliogenesis, have de novo missense variants in CDD probands: $B B S 5$ and $B B S 9$. Although the specific protein-changing variants identified in CDD subjects were rare and not previously associated with disease, we reviewed the literature and found some overlap between CDD candidate genes and genes potentially associated with other neurological disorders (Table 2).

There were no clearly deleterious variants in the CDD probands. To identify potentially pathogenic variants, we considered a combination of factors: (1) positive brain expression, (2) PhyloP score $\geq 1.30$ ( $P=0.05$ for conservation), (3) negative RVIS (gene intolerant of variation), and (4) PolyPhen-2 classification of probably damaging missense (or $\mathrm{n} / \mathrm{a}$ due to a variant other than missense). Of the $47 \mathrm{CDD}$ candidate genes, 14 met all of these criteria: NRK, TBC1D8B, TRRAP, NAV2, OGDHL, ZNF236, PRKCSH, MTMR8, BCOR, SRPK3, USP9Y, KIAA2018, CXorf57, and ALG13 (Table 2). To further refine this list, inspection of sequencing data from the Exome Aggregation Consortium (http://exac.broadinstitute.org/) revealed that: (1) the variants in all of the genes except NAV2, MTMR8, and ALG13 are novel or found at most once in the dataset and (2) among the remaining 11 genes, 4 are among the $5 \%$ most intolerant: TRRAP, ZNF236, BCOR, and KIAA2018.

TRRAP (transformation/transcription domain-associated protein)affected by a de novo missense variant in a male CDD proband; it encodes a component of histone acetyltransferase complexes and is involved in DNA transcription and repair. It is not associated with an OMIM disorder, but de novo variants have been identified in other neurological disorders (Table 2). ZNF236 (Zinc Finger Protein 236) is also affected by a de novo missense variant in a male proband; it may be involved in transcriptional regulation (UniProtKB) but is not associated with a known disorder. $B C O R$ ( $B C L 6$ Corepressor) is affected by a hemizygous missense variant in a male CDD proband; it encodes a transcriptional corepressor. It is associated with syndromic 


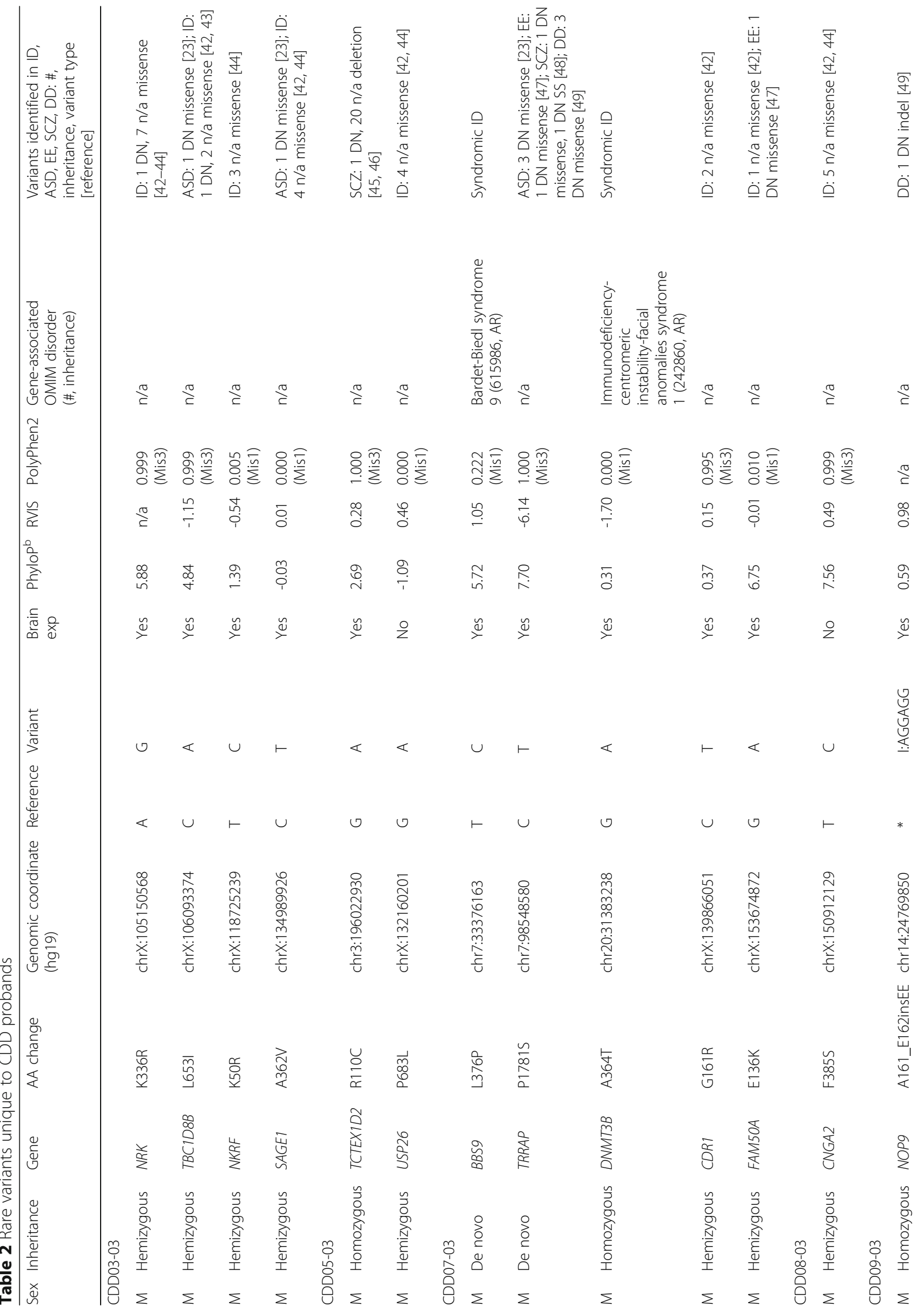




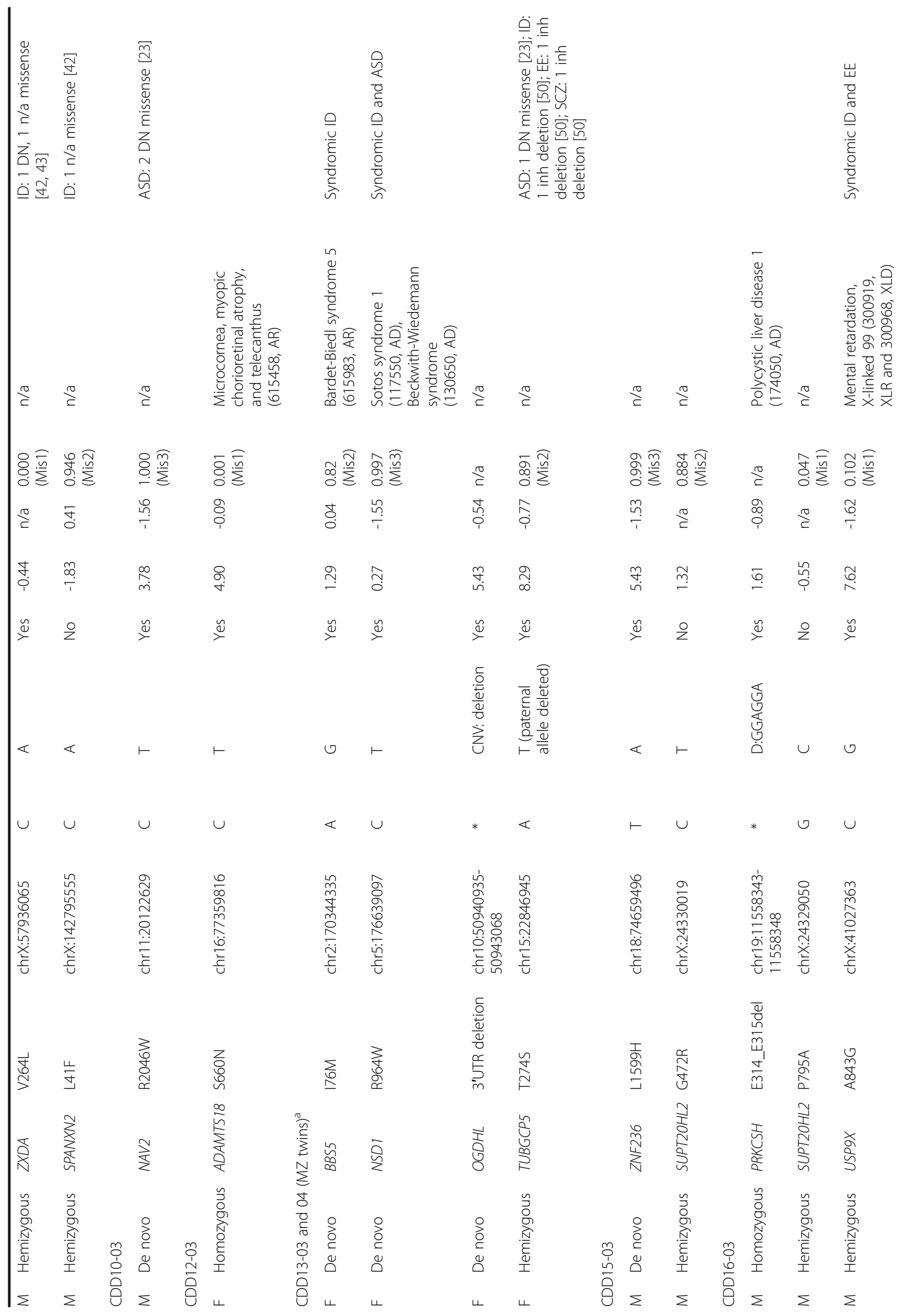



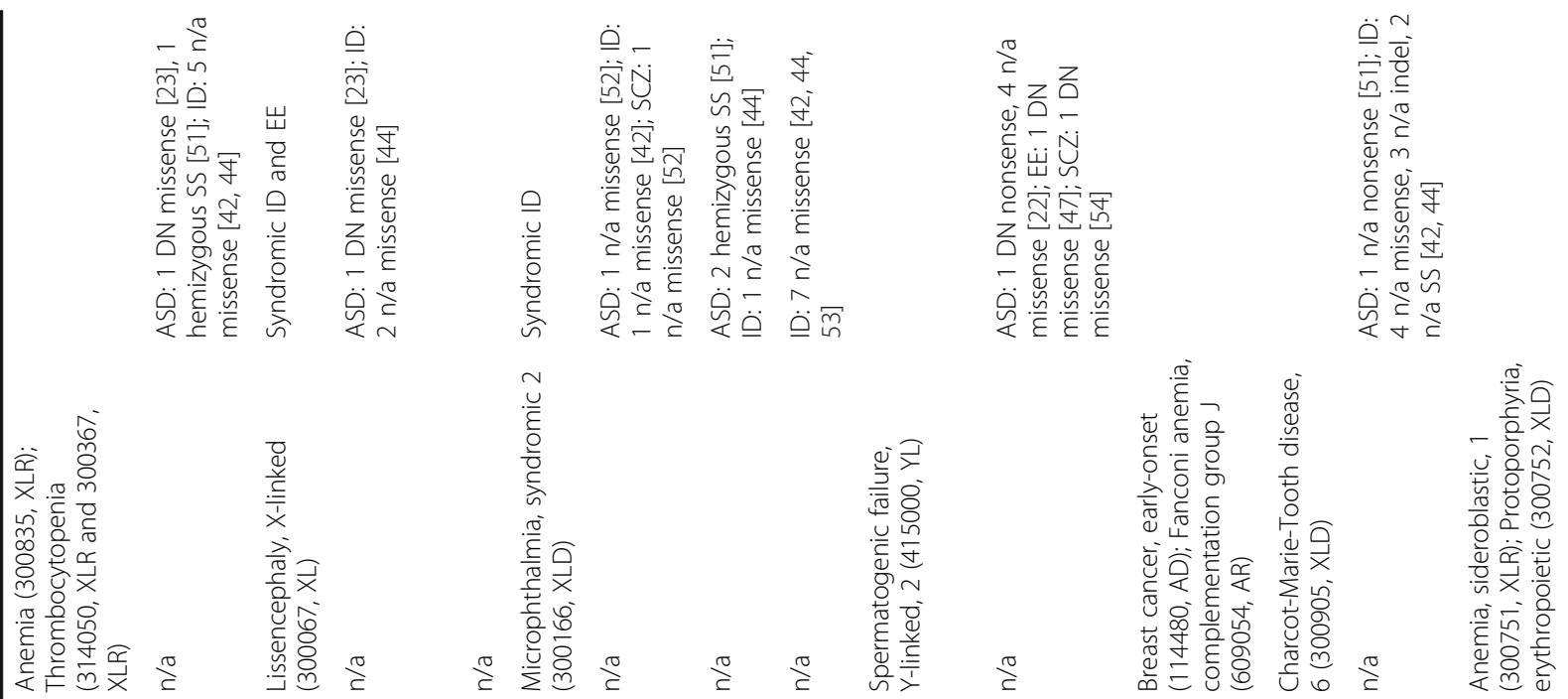

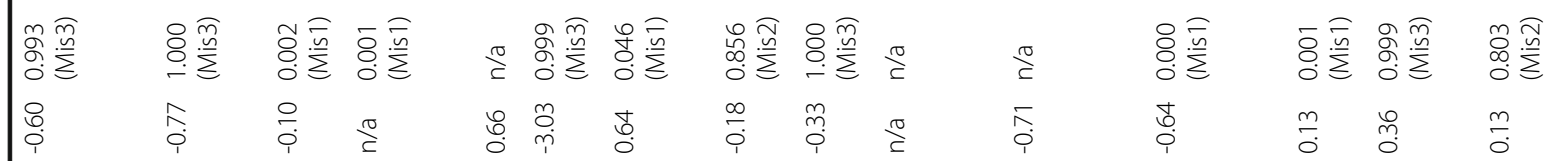

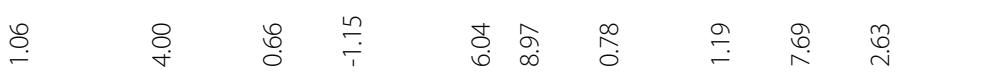

$\stackrel{\infty}{m}$

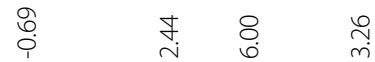

$\stackrel{Ð}{\rightleftharpoons}$

$\stackrel{\Perp}{\longleftarrow}$

$\stackrel{\Perp}{\rightleftharpoons} \stackrel{\circ}{\gtrless}$

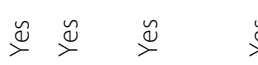

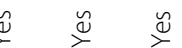

$\stackrel{y}{\check{1}}$

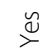

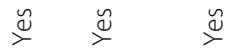

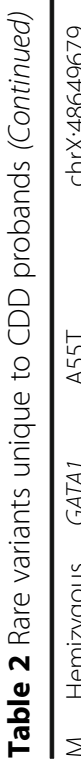

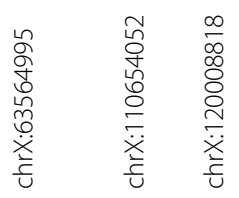

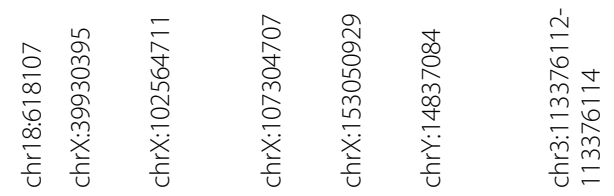

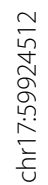

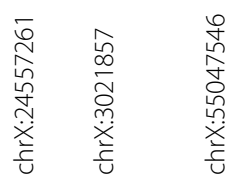

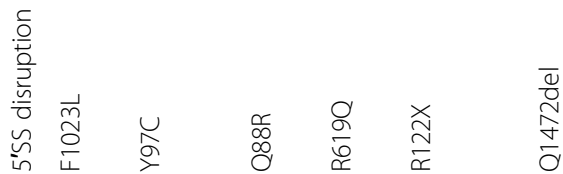

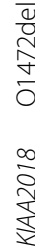

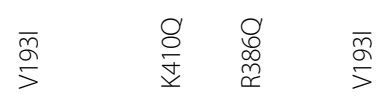

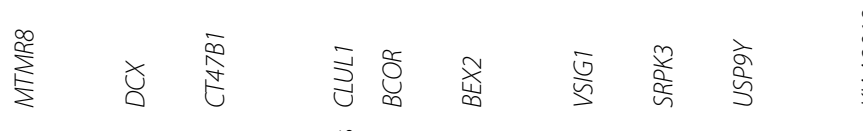

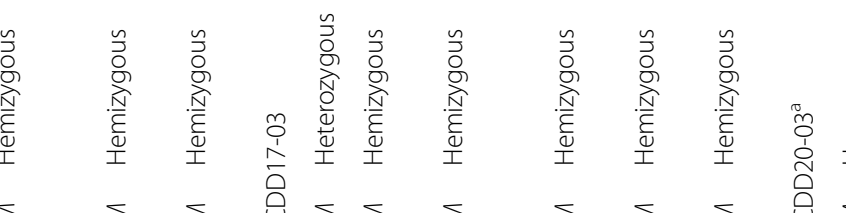

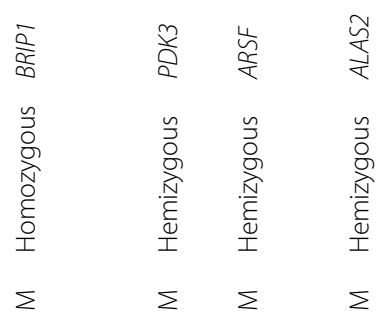




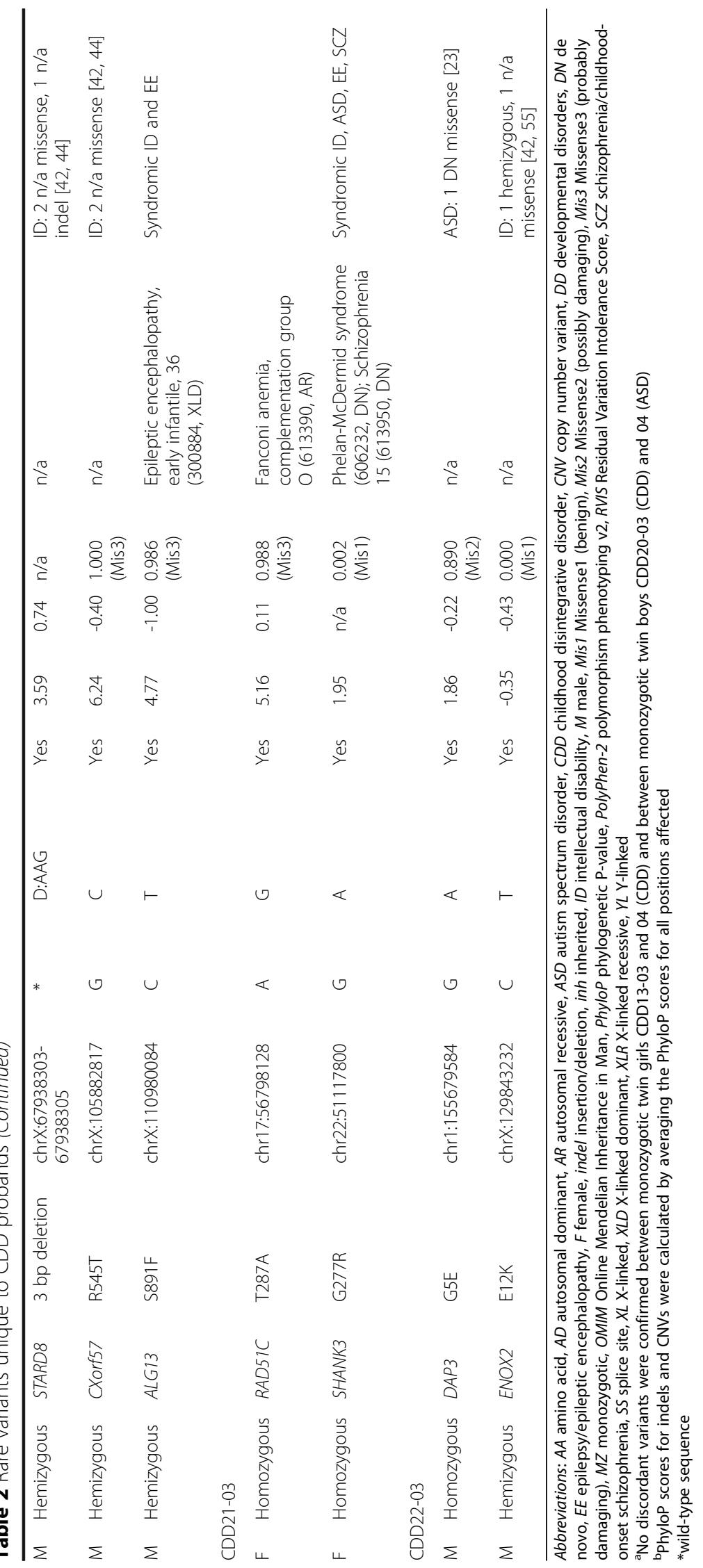


microphthalmia, which can have the feature of ID but otherwise does not characterize the CDD proband and is usually caused by truncating mutations in females. KIAA2018 is affected by a homozygous one-amino acid deletion in a male CDD proband; it is also known as USF3 (upstream transcription factor 3). It is not associated with an OMIM disorder, but de novo variants have been identified in other neurological disorders (Table 2). Of note, all of these top candidate genes either play a role or may be involved in transcription, which characterizes many ASDassociated genes as well [22].

Using the human BrainSpan exon-array transcriptome dataset [20], we plotted the median expression level of the CDD candidate genes as a group for all the brain regions available from embryonic to late adulthood stages ( $n=40$ genes represented once in the core probe set, Additional file 2: Table S4). As shown by the expression profile in Fig. 1, CDD candidate genes are more highly expressed in non-neocortical regions [hippocampus (HIP), amygdala (AMY), striatum (STR), mediodorsal nucleus of the thalamus (MD), and/or cerebellar cortex $(\mathrm{CBC})]$ compared to neocortical regions across the lifespan (Additional file 2: Table S5). Moreover, there are increasing levels of expression in the AMY, STR, and HIP during periods 10 (1-6 years old) and 11 (612 years-old), the range that encompasses the age of onset of symptoms in our CDD cohort.

Given this observation, we compared the difference in median expression levels between non-neocortical and neocortical regions for genes affected by nonsynonymous and synonymous variants in CDD probands, their unaffected siblings, and ASD probands from the Simons Simplex Collection (SSC) with and without regression [23] matched by sex, age at evaluation, IQ, and autism symptom severity (see Additional file 1: Supplementary information for cohort selection details). The expression profile of CDD candidate genes is qualitatively distinct from the other gene sets, except that it is similar to the profile of genes affected by nonsynonymous variants in SSC probands with regression, even though they have only one gene, $N A V 2$, in common (Fig. 2, Additional file 2 : Tables S4 and S6). The difference in expression, non-neocortical minus neocortical, reaches a maximum positive value at mid-fetal stages. For CDD candidate genes, this occurs at period six [19-24 postconceptual weeks (PCW)]; permutation testing with 100,000 iterations of 40 randomly selected genes from the BrainSpan dataset confirmed the significance of this differential expression $(P=0.0022)$. We extended the analysis to several other gene sets, such as those identified in SSC probands and unaffected siblings with non-synonymous, synonymous, and LGD variants; genes most significantly associated with ASD by three recent large WES and CNV studies [22-24]; and all genes in the BrainSpan dataset. The expression profile of genes affected by non-synonymous variants in CDD probands and SSC probands with regression is qualitatively distinct from these other sets as well (Additional file 1: Figure S1, Additional file 2: Tables S4 and S6).

We also investigated whether CDD candidate genes are coexpressed with each other. Of the 40 candidate genes, 11 are coexpressed with at least one other candidate gene across all brain regions and time periods with a Pearson correlation coefficient $r \geq 0.7$ (Fig. 3, Additional file 2: Table S7). There are 23 such connections, for a mean of 2.09 correlations/gene and a mean coefficient of 0.779 . Permutation testing with 100,000 iterations of 40 randomly selected genes from the BrainSpan dataset revealed that observing 11 genes with at least 2.09 correlations/gene is significant $(P=$ 0.036), as is observing 11 genes with a mean correlation coefficient of at least $0.779(P=0.019)$. Meeting both thresholds is also significant $(P=0.0059)$. Since all 11 CDD candidate genes which are coexpressed with each other have positive brain expression as per the BrainSpan dataset, permutation testing with 100,000 iterations was also performed with 40 randomly selected brain-expressed genes from BrainSpan. While observing 11 genes with at least 2.09 correlations/gene is not significant $(P=0.066)$, observing 11 genes with a mean correlation coefficient of at least 0.779 is significant $(P=$ $0.022)$ as is meeting both thresholds $(P=0.011)$. Comparing the set of 11 coexpressed CDD candidate genes with the remaining set of 29 which are not coexpressed revealed no significant differences between the rate of brain-expressed genes, PhyloP scores, or PolyPhen-2 scores; however, the coexpressed genes are significantly more intolerant of variation (average RVIS -1.42 versus $-0.15, t(35)=-2.91, P=0.0062$, independent $t$ test, twotailed). Gene ontology enrichment analysis using the Database for Annotation, Visualization and Integrated Discovery v6.8 (https://david.ncifcrf.gov/) for the whole set of CDD candidate genes, and the subset of 11 coexpressed genes did not identify significant enrichment of GO terms after Benjamini-Hochberg correction of $P$ values.

\section{Neural systems}

Given the universality of social deficits in ASD, dysfunction in brain systems subserving social perception, including the perception of faces, is a key focus of ASD research. Face and house visual stimuli reliably activate and dissociate systems involved in socioemotional (fearful faces) and non-socioemotional (houses) information processing. We studied four cohorts: CDD $(n=7)$, LFASD $(n=7)$, HFASD $(n=14)$, and TD $(n=19)$. Even though individuals with LFASD are more numerous than those with CDD, our sample size was still limited by the difficulty of obtaining high-quality neuroimaging (and 


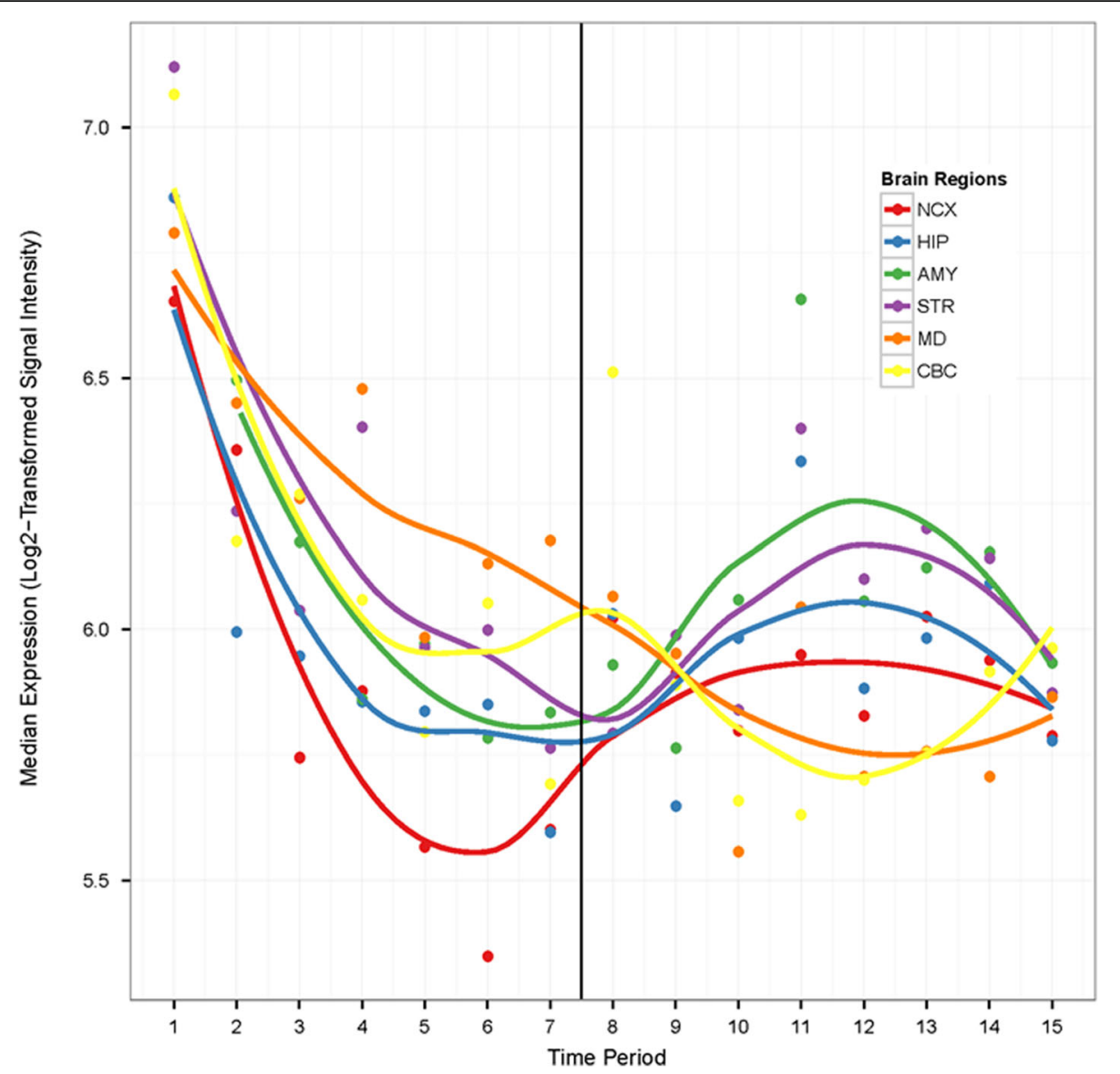

Fig. 1 Median expression levels of CDD candidate genes $(n=40)$ by brain region and time period (Additional file 2: Table S5) using the human BrainSpan exon-array transcriptome dataset [20]. The dark vertical line indicates birth. $\log _{2}$-transformed signal intensity $\geq 6$ in at least one sample is considered positive expression [20]. AMY amygdala, CBC cerebellar cortex, HIP hippocampus MD mediodorsal nucleus of the thalamus, NCX neocortex, STR striatum

eye-tracking) data in low-functioning subjects. That being said, to our knowledge, this is the first ever presentation of non-sedated fMRI data from individuals with ASD and marked ID.

There were no significant differences in sex, age, intracranial volume, and head movement in the scanner between the four cohorts. The CDD and LFASD groups were also not significantly different by IQ and autism severity, and the HFASD and TD groups were not significantly different by IQ (Additional file 2: Tables S8 and S9). First, we utilized a discovery sample of 12 of our 19 TD subjects in a whole-brain analysis for an independent localization of regions of interest involved in processing faces relative to houses. Figure 4a illustrates regions of ventrolateral occipitotemporal cortex where TD subjects exhibited significant faces $>$ houses activation (Additional file 2: Table S10). These regions included the expected locations of well-known nodes of the occipitotemporal face-sensitive network including the fusiform face area $[28,29]$ and the occipital face area
[30]. As shown in Fig. 4b and Additional file 2: Table S11, extraction of the mean percent signal change (faces $>$ houses) for each of the four groups [TD:validation (the remaining 7 of the 19 TD subjects), HFASD, LFASD, and CDD] indicated an absence of group differences in the response to faces versus houses in these independently defined regions of interest when comparing the TD:validation and HFASD groups $[t(19)=0.17, P=$ 0.87, Cohen's $d=0.08$ ] and when comparing the LFASD and CDD groups $[t(12)=0.97, P=0.35$, Cohen's $d=0.56]$. The faces $>$ houses response within the CDD group was not significantly greater than zero $[t(6)=$ $0.80, P=0.45$, Cohen's $d=0.30]$, suggesting an overall lack of sensitivity to faces in the occipitotemporal facesensitive network as a whole. There is a wellestablished finding of hypoactivation to faces (versus houses) in the right, middle fusiform gyrus in HFASD relative to TD [31]. We were able to replicate this finding in our cohorts $[t(31)=3.54, P=0.0013$, Cohen's $d$ $=1.29$ ]. However, comparison of faces $>$ houses activity 


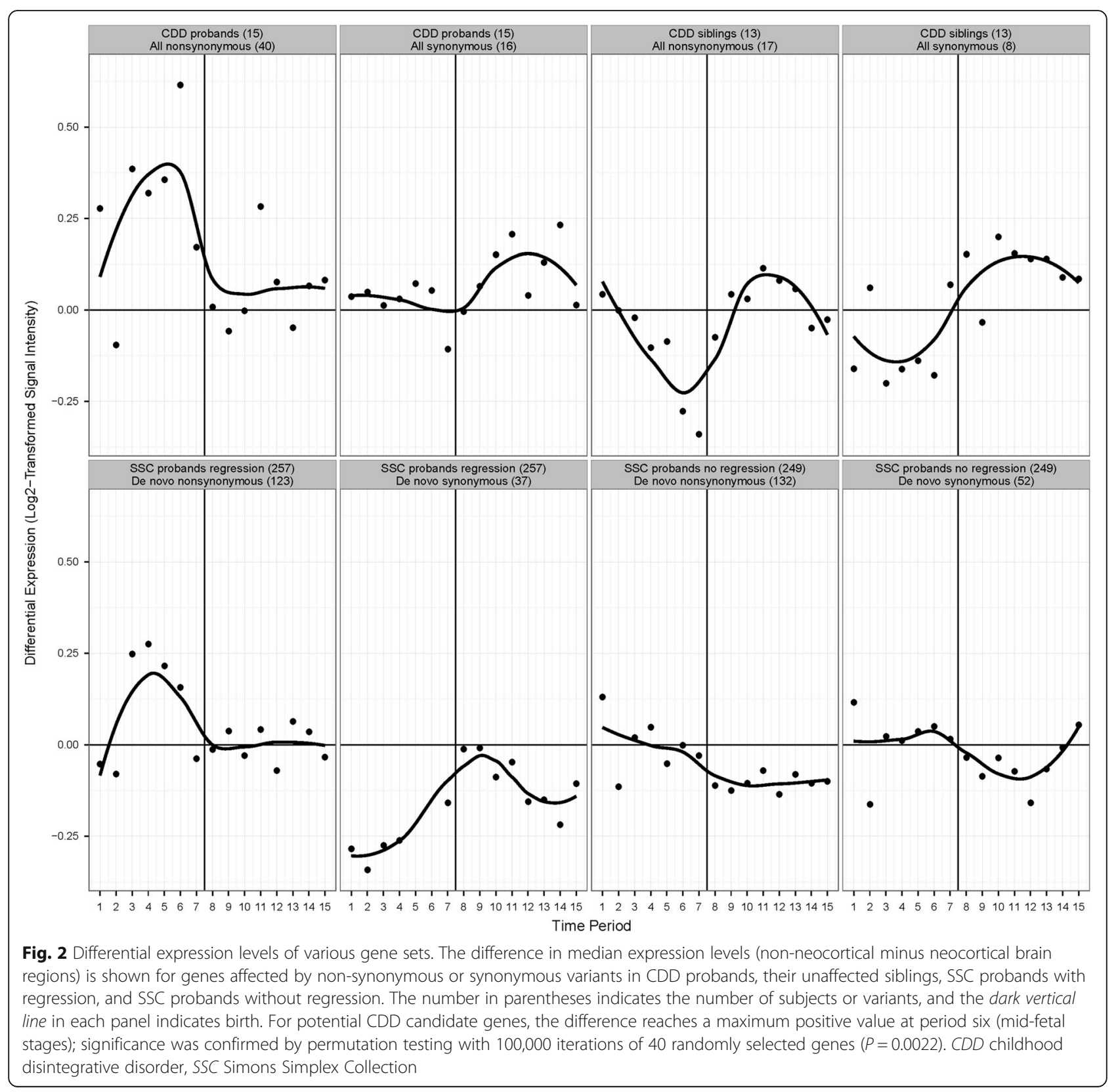

in CDD relative to TD revealed no significant difference $[t(24)=1.18, P=0.25$, Cohen's $d=0.54]$, as did the comparison of the LFASD and TD groups $[t(24)=1.10$, $P=0.28$, Cohen's $d=0.51$ ] (Additional file 1: Figure S2 and Additional file 2: Table S12).

Given the possible lack of sensitivity to faces in the ventrolateral occipitotemporal cortex in $\mathrm{CDD}$, we next conducted a whole-brain evaluation of the CDD subjects to localize the neuroanatomical substrates of face perception in these individuals. As illustrated in Fig. 5a, CDD subjects exhibited faces $>$ houses activity in the middle frontal gyrus, precentral gyrus, caudate (striatum), thalamus, hippocampus, and cerebellum (Additional file 2: Table S13). These overlap with brain regions determined to have the highest levels of CDD candidate gene expression (Fig. 1). As shown in Fig. 5b and Additional file 2: Table S14, comparison of the mean percent signal change (faces $>$ houses) from these regions of interest revealed a significant difference between CDD and HFASD $[t(19)=2.98, P=0.0076$, Cohen's $d=1.45]$, but no significant difference between CDD and LFASD $[t(12)=$ 1.71, $P=0.11$, Cohen's $d=0.99$ ]. The LFASD group showed an intermediate phenotype to that of HFASD and CDD groups (Fig. 5b). 


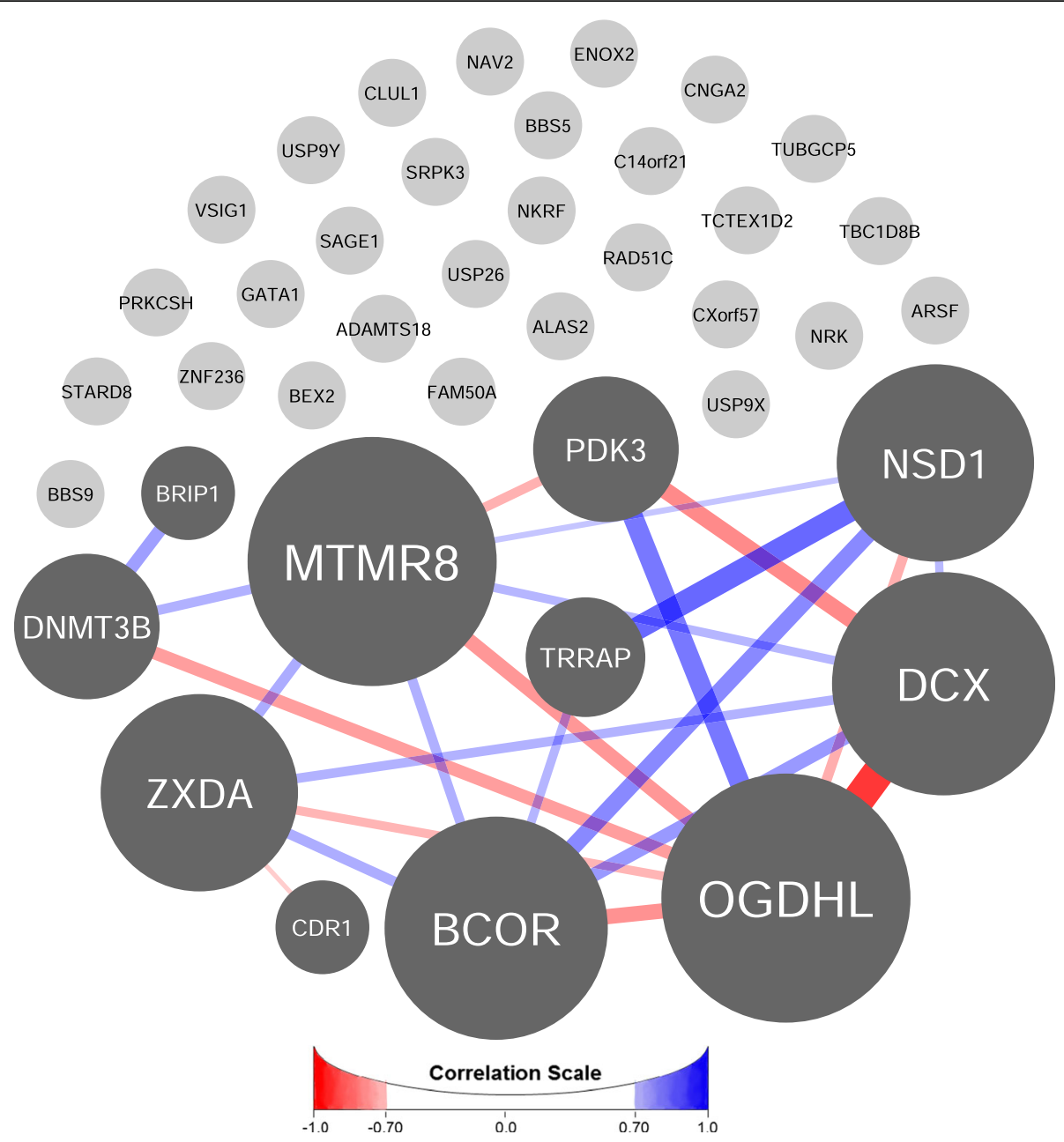

Fig. 3 Gene coexpression network analysis. Eleven of the 40 CDD candidate genes are coexpressed with at least one other candidate gene across all brain regions and time periods with a Pearson correlation coefficient $r \geq 0.7$ (Additional file 2: Table S7), a mean of 2.09 correlations/ gene $(P=0.036)$, and a mean coefficient of $0.779(P=0.019$, permutation testing with 100,000 iterations of 40 randomly selected genes). Positive correlations are shown in blue, and negative correlations are shown in red. The greater the magnitude of the coefficient, the wider and darker are the edges. The size of a node is proportional to the number of edges the node has

\section{Eye-gaze behavior}

We collected eye-tracking data to quantify the social phenotype of our four cohorts [CDD $(n=5)$, LFASD $(n=7)$, HFASD $(n=32)$, and TD $(n=14)]$ as they viewed emotional faces [21]. As shown in Additional file 2: Tables S15 and S16, the groups were not significantly different by sex, age, and total fixation duration on the image. The CDD and LFASD groups were also not significantly different by IQ and autism severity, and the HFASD and TD groups were not significantly different by IQ. As shown in Fig. 6, we replicate prior findings [32-34] of decreased fixation on the eyes $[t(44)=-2.28, P=0.03$, Cohen's $d=0.77]$ and increased fixation on the mouth $[t(44)=2.16, P=0.04$, Cohen's $d=0.76]$ in HFASD relative to TD. However, while the percentage of time subjects with LFASD spent looking at the eyes did not differ significantly from the HFASD group $[t(37)=0.43, P=0.67$, Cohen's $d=0.17]$, CDD subjects fixated eyes significantly more than the HFASD group [ $t(35)=2.19, P=0.04$, Cohen's $d=1.08$ ] Compared to each other, CDD and LFASD subjects did not differ significantly in time spent looking at the eyes $[t(10)=1.35, P=0.21$, Cohen's $d=0.87]$. As with the fMRI results (Fig. 5b), the LFASD group showed an intermediate phenotype to that of the HFASD and CDD groups (eye-mouth ratio, Additional file 2: Table S16).

\section{Discussion}

We are reporting the first multimodal analysis of CDD, a rare form of ASD characterized by late-onset, severe regression. The small cohort size due to its low prevalence, and the challenges of obtaining interpretable data from non-sedated fMRI and eye tracking in subjects with ASD and significant ID necessitated an exploratory study. 

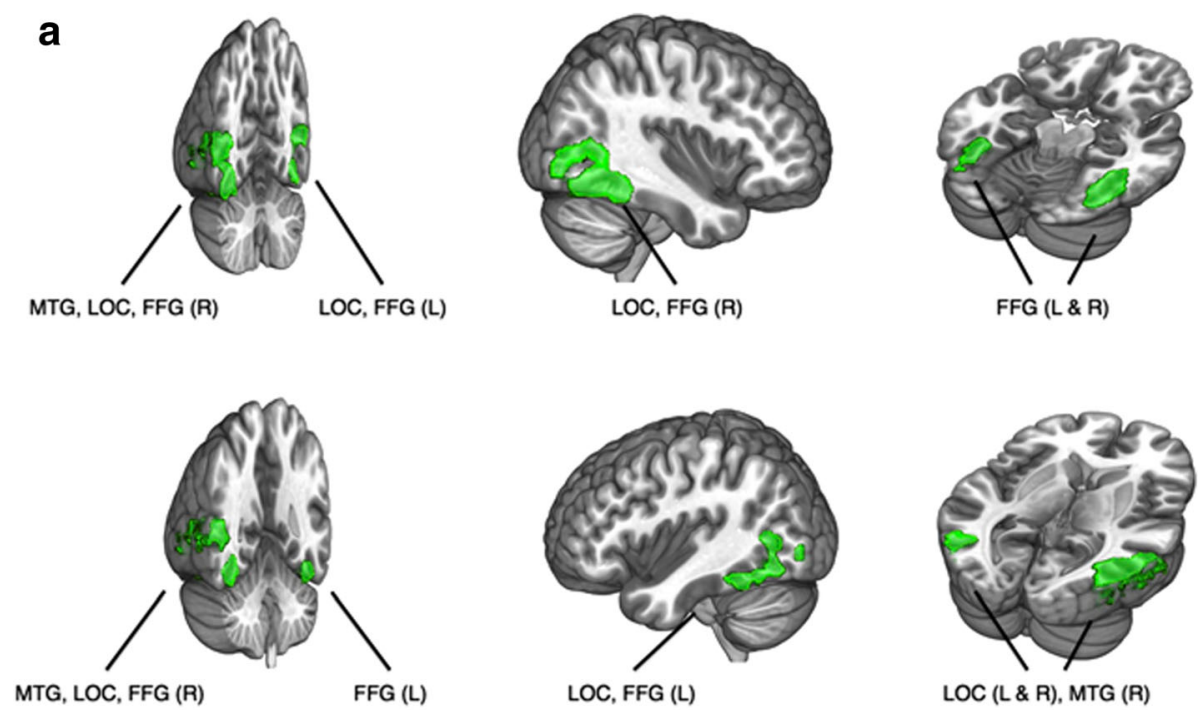

LOC (L\& R), MTG (R)

b

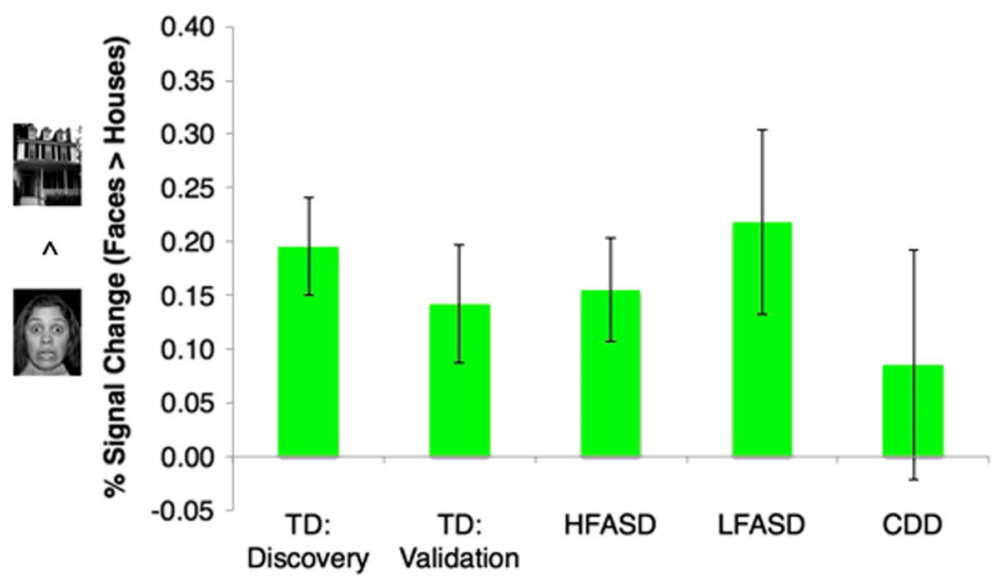

Fig. 4 Brain regions of interest (ROIs) involved in processing socioemotional (fearful face) versus non-socioemotional (house) visual stimuli. a The green color brain map indicates regions of significant faces $>$ houses activation in a discovery sample of 12 TD subjects $(Z>3.09$, whole-brain corrected at the cluster-level $P<0.05)$. $\mathbf{b}$ These independently defined ROls were then utilized for comparisons across the four remaining cohorts, a TD:validation sample $(n=7)$, HFASD $(n=14)$, LFASD $(n=7)$, and CDD $(n=7)$. The bar graph indicates the mean \% signal change (faces $>$ houses) for each cohort. Group differences were not significant when comparing the TD:validation and HFASD groups [t(19) $=0.17, P=0.87$, Cohen's $d=0.08]$ and when comparing the LFASD and CDD groups $[t(12)=0.97, P=0.35$, Cohen's $d=0.56]$. The faces $>$ houses response within the CDD group was not significantly greater than zero $[t(6)=0.80, P=0.45$, Cohen's $d=0.30]$. Error bars indicate standard error of the mean. All $P$ values were calculated by independent $t$ test and are two-tailed. FFG fusiform gyrus, L left, LOC lateral occipital cortex, MTG middle temporal gyrus, $R$ right

There is a relative deficiency of reports using these protocols with low-functioning individuals on the autism spectrum. For the first time, a combination of fMRI and eye-tracking analyses are being presented for such individuals to help fill this gap.

Gene expression, neuroimaging, and social behavior analyses suggest that there are neurobiological differences which may underlie the distinct clinical features of CDD. Although no clearly deleterious variants or highly recurrent candidate genes were identified, candidate genes most conserved at variant position or most intolerant of variation, such as TRRAP, ZNF236, and KIAA2018, play a role or may be involved in transcription, which characterizes many ASD-associated genes as well [22]. Gene expression analysis provided some potential insights into CDD. The expression profile of CDD candidate genes resembled that of SSC probands with regression but not SSC probands without regression (matched by IQ), suggesting a pattern relevant to regression. A significant number of CDD candidate genes are co-expressed and may interact in pathways important to the pathophysiology of the disorder. 
a

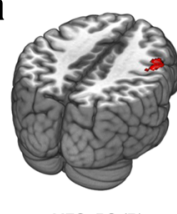

MFG, PG (R)

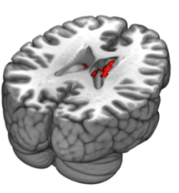

Caudate (R)

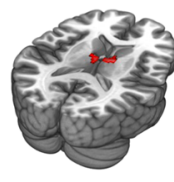

Thalamus (L\& R)

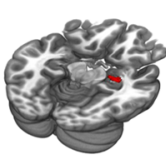

Hippocampus (R)

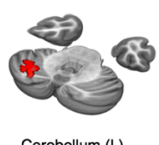

Cerebellum (L)

b

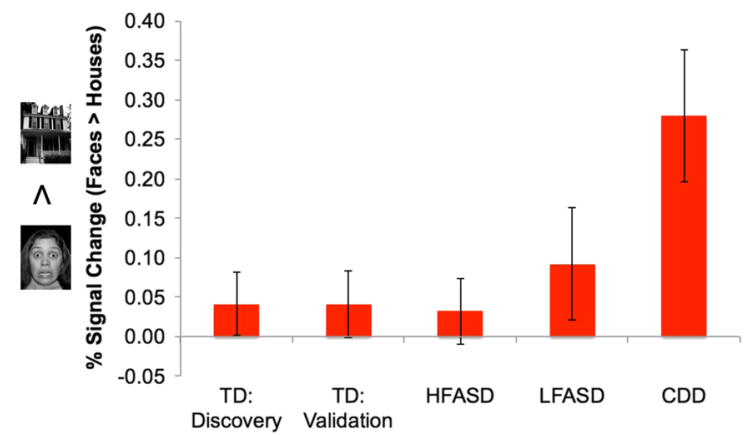

Fig. 5 CDD whole-brain fMRI analysis. a The red color brain map indicates regions of significant faces $>$ houses activation in the CDD subjects ( $Z>3.09$, whole-brain corrected at the cluster-level $P<0.05)$. b The bar graph indicates the mean \% signal change (faces $>$ houses) within these areas for each cohort: TD:discovery $(n=12)$, TD:validation $(n=7)$, HFASD $(n=14)$, LFASD $(n=7)$, and CDD $(n=7)$. The CDD cohort differed significantly from HFASD $[t(19)=2.98, P=0.0076$, Cohen's $d=1.45]$ but not from LFASD [t(12) $=1.71, P=0.11$, Cohen's $d=0.99]$. Error bars indicate standard error of the mean. All $P$ values were calculated by independent $t$ test and are two-tailed. MFG middle frontal gyrus, $P G$ precentral gyrus

Interestingly, expression of the candidate genes overlapped with face-evoked brain hyperactivity in CDD in non-neocortical regions, such as the thalamus, cerebellum, caudate (striatum), and hippocampus. These regions are known to be involved in distributing eye movements (and thus attention) to socially meaningful stimuli, including faces, early in development. Increased face-evoked activity in CDD was paralleled by increased attention to the eyes of faces, culminating in a normal distribution of attention to the eyes. Still unresolved,

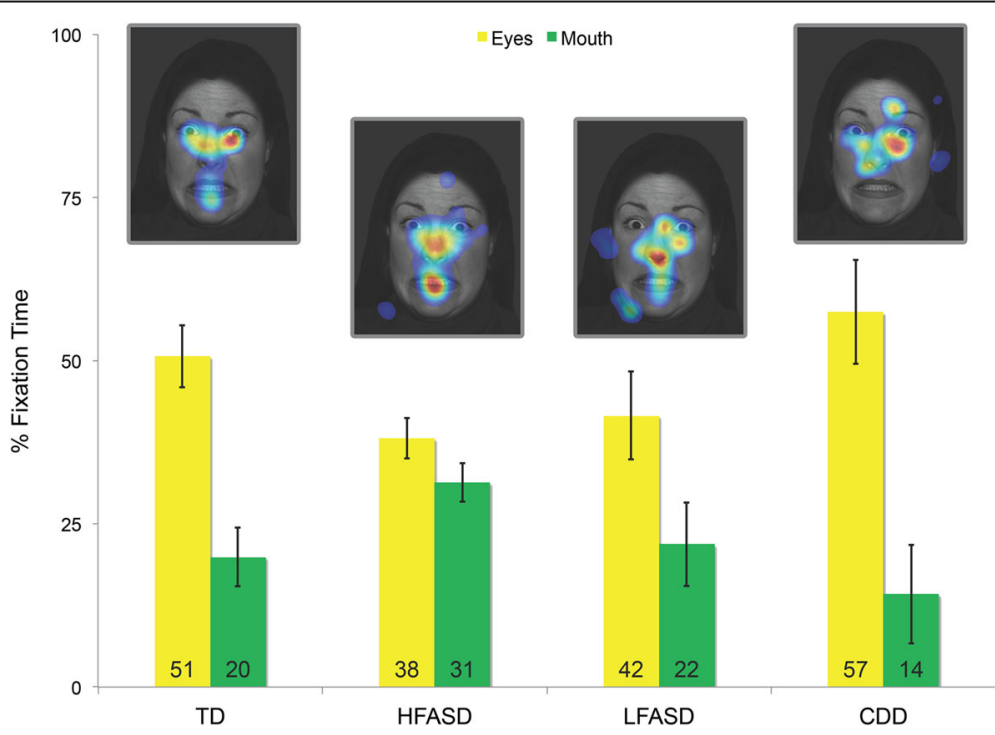

Fig. 6 Behavioral analysis through eye tracking. The yellow and green bars of the graph represent the mean \% of time spent fixating (y axis) on the eyes and mouth of the faces, respectively, by cohort ( $x$ axis): TD $(n=14), \operatorname{HFASD}(n=32), \operatorname{LFASD}(n=7), \operatorname{CDD}(n=5)$. The gaze heat maps illustrate the group-level gaze data overlaid on one of the images at which subjects looked. Compared to TD subjects, HFASD subjects show decreased fixation on the eyes $[t(44)=-2.28, P=0.03$, Cohen's $d=0.77]$ and increased fixation on the mouth $[t(44)=2.16, P=0.04$, Cohen's $d=0.76]$. The \% of time subjects with LFASD spent looking at the eyes did not differ from HFASD [t(37) $=0.43, P=0.67$, Cohen's $d=0.17]$, but CDD subjects fixated eyes significantly more than HFASD $[t(35)=2.19, P=0.04$, Cohen's $d=1.08]$. Error bars indicate standard error of the mean. All $P$ values were calculated by independent $t$ test and are two-tailed 
though, is how a more typical viewing pattern relates to the poor outcomes which characterize CDD.

Individuals with ASD with greater communicative competence show a more atypical pattern of attention toward faces comprised of decreased looking at the eyes and increased looking at the mouth $[35,36]$, while individuals with ASD and language impairment have been reported to not differ from typical peers [36]. We found a similar discontinuity in face information processing behaviors, with atypical face-viewing strategies evidenced most clearly for the (most able) HFASD group, and a more typical pattern for the CDD group. While CDD did not differ significantly from LFASD on eye-tracking measures, CDD showed the strongest between group differences in effect sizes referenced against the atypical looking patterns of HFASD. The presence of intact orientation to the eyes and unusual face-sensitive brain activation suggest an alternative developmental pathway for face processing in CDD.

Coinciding with the onset of canonical babbling, the typical infant's transition from looking at the eyes of a speaker to looking at the mouth is between 4 and 8 months of age [37]. This bias reverts back to the eyes by 12 months for infants viewing people speaking their native language (but not a foreign language), an effect probably driven by advancing expertise and perceptual narrowing. Preferences for looking at mouths in HFASD may reflect higher-order cognitive compensatory mechanisms with scaffolding functions analogous to the 48 month transitional period in typical development or biases for second languages later in infancy [33, 38], whereas LFASD and CDD may lie on opposite sides of the 4-8 month divide. The unique face-evoked activity that localized to a set of subcortical structures and the cerebellum in CDD suggests a neoteny in the development of the face-processing system whereby subcortical mechanisms thought to control orienting and attention to faces $[39,40]$ early in human development remain abnormally involved or cease to be inhibited by top-down regulation following regression. This may represent a marker of the unique developmental process underlying CDD, thereby suggesting a target for studies utilizing eye tracking for early identification and stratification of behaviorally and biologically heterogeneous forms of ASD $[36,41]$.

It is important to note that our investigations occurred months to years after the onset of symptoms. Since CDD is typically a diagnosis of exclusion, subjects come to our attention for the purposes of research long after the regressive period. Therefore, how the neurobiological features of CDD that we identified relate to the course of regression is unknown. It will be essential to confirm our results in larger cohorts. Ideally, subjects with CDD would be studied before and after the regression to better identify neurobiological correlates; however, this is challenging with such a rare disorder. Since regression is frequently described in ASD, prospective studies of more typical cases of regression may determine whether our results are relevant to regression in the autism spectrum more broadly. It would also be interesting to conduct our studies in regressive disorders such as Rett syndrome. Furthermore, since the fMRI and eye-tracking results revealed that the LFASD subjects had phenotypes intermediate to those of CDD and HFASD, it will be important to study an ID cohort without ASD to better attribute group differences to the effects of ASD versus ID. A major future challenge will be to elucidate the mechanisms by which variants in a set of genes may lead to areas of brain hyperactivity and an apparently normal attention to eyes but, in the end, the severe autism which characterizes CDD.

\section{Conclusions}

In summary, we pursued a multidisciplinary, multi-level approach comprising genetic, brain, and behavioral analyses to conduct an exploratory study of CDD, a rare and severe condition of unclear etiology. Although CDD and other forms of ASD have clinical similarities, the unique natural history of CDD may mark some unique neurobiological features. The clinical and genetic heterogeneity of ASD are well established; our results suggest that there is also heterogeneity of biomarkers, such as affected brain regions and neural circuits. Biomarkers established for high-functioning individuals with ASD may not apply to the substantial proportion of individuals on the spectrum who have ID. Ultimately, the recognition of an increasing number of specific ASD biotypes may translate into more targeted diagnostic tests and treatments.

\section{Additional files}

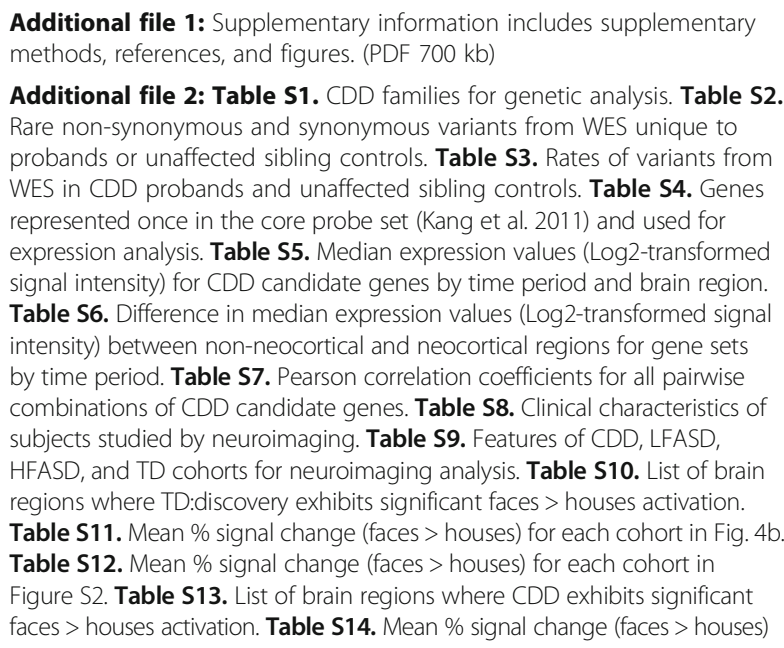

Additional file 2: Table S1. CDD families for genetic analysis. Table S2. Rare non-synonymous and synonymous variants from WES unique to probands or unaffected sibling controls. Table S3. Rates of variants from WES in CDD probands and unaffected sibling controls. Table S4. Genes represented once in the core probe set (Kang et al. 2011) and used for expression analysis. Table S5. Median expression values (Log2-transformed signal intensity) for CDD candidate genes by time period and brain region. Table S6. Difference in median expression values (Log2-transformed signal intensity) between non-neocortical and neocortical regions for gene sets by time period. Table S7. Pearson correlation coefficients for all pairwise combinations of CDD candidate genes. Table S8. Clinical characteristics of subjects studied by neuroimaging. Table S9. Features of CDD, LFASD, HFASD, and TD cohorts for neuroimaging analysis. Table S10. List of brain regions where TD:discovery exhibits significant faces $>$ houses activation. Table S11. Mean \% signal change (faces $>$ houses) for each cohort in Fig. 4b. Table S12. Mean \% signal change (faces $>$ houses) for each cohort in Figure S2. Table S13. List of brain regions where CDD exhibits significant faces $>$ houses activation. Table S14. Mean \% signal change (faces $>$ houses) 
for each cohort in Fig. 5b. Table S15. Clinical characteristics of subjects studied by eye tracking. Table S16. Features of CDD, LFASD, HFASD, and TD cohorts in eye-tracking analysis. Table S17. Whole-exome sequencing quality metrics. Table S18. Features of SSC probands with and without regression by IQ and autism severity. (XLSX $364 \mathrm{~kb}$ )

\section{Abbreviations}

AMY: Amygdala; ASD: Autism spectrum disorder; CBC: Cerebellar cortex; CDD: Childhood disintegrative disorder; CNV: Copy number variant; FFG: Fusiform gyrus; fMRl: Functional magnetic resonance imaging; FSIQ: Full-scale IQ; HFASD: High-functioning ASD; HIP: Hippocampus; ID: Intellectual disability; IQ: Intelligence quotient; LFASD: Low-functioning ASD; LGD: Likely gene-disrupting; LOC: Lateral occipital cortex; MD: Mediodorsal nucleus of the thalamus; MFG: Middle frontal gyrus; MTG: Middle temporal gyrus; OMIM: Online Mendelian Inheritance in Man; PG: Precentral gyrus; PhyloP: Phylogenetic $P$ value; PolyPhen-2: Polymorphism Phenotyping v2; ROI: Region of interest; SSC: Simons Simplex Collection; STR: Striatum; TD: Typically developing; WES: Whole-exome sequencing

\section{Acknowledgements}

We are very grateful to the families who participated in this study. We are thankful to the following colleagues for assistance and comments: Kaya Bilguvar, Marie Claudel, Jessica Connelly, Charles Farber, Richard Lifton, Francesc Lopez, Shrikant Mane, John Murdoch, Laura Politte, Stephan Sanders, Ariella Ritvo Slifka, Michele Spencer-Manzon, Matthew State, Irina Tikhonova, Jeremy Willsey, Nicole Wright, and Andrew Zimmerman.

\section{Funding}

This research was funded by the Simons Foundation (206929 R10981 to ARG and KAP), the National Institutes of Health (K08MH087639 to ARG; K08MH099424-02 to TVF; Clinical Investigation and the Clinical and Translational Science UL1 TR000142 and KL2 TR000140 from the National Center for Research Resources to AGE-S; CTSA UL1 RR024139, Expedition in Computing award 1139078, and R21MH102572 to FS; RR19895 and RR029676-01 to the Yale University Biomedical High Performance Computing Center), the Alan B. Slifka Foundation and Foundation Rumsey Cartier to AW, and Autism Speaks Meixner Postdoctoral Fellowship in Translational Research (\#9284) and Hilibrand Autism Fellowship to DYJY.

\section{Availability of data and materials}

The data generated and analyzed during this study are included in this article (and its additional files) and are available from the corresponding author upon request.

\section{Authors' contributions}

ARG conceived and designed the genetics study, collected and analyzed the genetic data, evaluated the medical records, and co-authored the manuscript. AW recruited and performed clinical characterization of the study subjects, co-designed the fMRI and eye-tracking experiments, and collected and analyzed the fMRI and eye-tracking data. DYJY analyzed the fMRI data. CAWS collected and analyzed WES and CNV data. JE analyzed fMRI and eye-tracking data. SZ collected and analyzed WES data. AV collected and analyzed fMRI and eyetracking data. BCWW analyzed fMRI data. PV performed clinical characterization of study subjects. ZW collected and analyzed WES and CNV data. TVF analyzed genotyping data and performed ancestry mapping. AGES analyzed and confirmed CNV data. MFW collected and analyzed WES data. MC collected and analyzed WES data. AS analyzed fMRI data. TH recruited and performed the clinical characterization of study subjects. GB recruited and performed clinical characterization of study subjects. HF analyzed clinical characteristics of study subjects and managed fMRI and eye-tracking databases. CC recruited and performed clinical characterization of study subjects. AR analyzed the clinical characteristics of study subjects. FS analyzed eye-tracking data. FRV recruited and performed the clinical characterization of study subjects. KAP conceived and designed the overarching gene-brain-behavior approach, co-designed the fMRI and eye-tracking experiments, and co-authored the manuscript. All authors read, edited, and approved the final manuscript.

\section{Competing interests}

The authors declare that they have no competing interests.

\section{Consent for publication}

The HIC protocol provides for consent for publication. However, no individually identifying information is presented in this report.

\section{Ethics approval and consent to participate}

This research was approved by the Yale University Institutional Review Board, and informed consent was obtained for all subjects (Human Investigations Committee (HIC) Protocol \# 0301024156 and 1004006656).

\section{Publisher's Note}

Springer Nature remains neutral with regard to jurisdictional claims in published maps and institutional affiliations.

\section{Author details}

'Department of Pediatrics, Yale School of Medicine, New Haven, Connecticut, USA. ${ }^{2}$ Child Study Center, Yale School of Medicine, New Haven, Connecticut, USA. ${ }^{3}$ Department of Psychiatry, Yale School of Medicine, New Haven, Connecticut, USA. ${ }^{4}$ Department of Genetics, Yale School of Medicine, New Haven, Connecticut, USA. ${ }^{5}$ Evelina London Children's Hospital, Guy's and St. Thomas' Trust, Kings Health Partners AHSC, London, UK.

Received: 22 December 2016 Accepted: 15 March 2017 Published online: 04 April 2017

\section{References}

1. American Psychiatric Association. Autism spectrum disorder. In: Diagnostic and Statistical Manual of Mental Disorders: DSM-5. 5th ed. Washington: American Psychiatric Association; 2013. p. 50-9.

2. Kanner L. Autistic disturbances of affective contact. Nerv Child. 1943;2:217-50.

3. Heller T. Dementia infantilis. Zeitschrift fur die Erforschung und Behandlung des Jugenlichen, Schwachsinns. 1908;2:17-28.

4. Westphal A, Schelinski S, Volkmar F, Pelphrey K. Revisiting regression in autism: Heller's dementia infantilis. J Autism Dev Disord. 2013;43:265-71.

5. World Health Organization. The ICD-10 classification of mental and behavioural disorders: clinical descriptions and diagnostic guidelines. Geneva: World Health Organization; 1992.

6. American Psychiatric Association. Pervasive Developmental Disorders. In: Diagnostic and statistical manual of mental disorders: DSM-IV. 4th ed. Washington: American Psychiatric Association; 1994. p. 65-71.

7. Hendry CN. Childhood disintegrative disorder: should it be considered a distinct diagnosis? Clin Psychol Rev. 2000;20:77-90.

8. Rosman NP, Bergia BM. Childhood disintegrative disorder: distinction from autistic disorder and predictors of outcome. J Child Neurol. 2013;28:1587-98.

9. Mouridsen SE, Rich B, Isager T. A comparative study of genetic and neurobiological findings in disintegrative psychosis and infantile autism. Psychiatry Clin Neurosci. 2000;54:441-6.

10. Volkmar FR, Cohen DJ. Disintegrative disorder or "late onset" autism. J Child Psychol Psychiatry. 1989:30:717-24

11. Volkmar FR, Rutter M. Childhood disintegrative disorder: results of the DSMIV autism field trial. J Am Acad Child Adolesc Psychiatry. 1995;34:1092-5.

12. Fombone E. Prevalence of childhood disintegrative disorder. Autism. 2002;6: 149-57.

13. Malhotra S, Gupta N. Childhood disintegrative disorder: re-examination of the current concept. Eur Child Adolesc Psychiatry. 2002;11:108-14.

14. Kurita $\mathrm{H}$, Osada $\mathrm{H}$, Miyake $\mathrm{Y}$. External validity of childhood disintegrative disorder in comparison with autistic disorder. J Autism Dev Disord. 2004;34: 355-62.

15. Homan KJ, Mellon MW, Houlihan D, Katusic MZ. Brief report: childhood disintegrative disorder: a brief examination of eight case studies. J Autism Dev Disord. 2011;41:497-504.

16. Barger BD, Campbell JM, McDonough JD. Prevalence and onset of regression within autism spectrum disorders: a meta-analytic review. J Autism Dev Disord. 2013:43:817-28.

17. Kurita H, Koyama T, Setoya Y, Shimizu K, Osada H. Validity of childhood disintegrative disorder apart from autistic disorder with speech loss. Eur Child Adolesc Psychiatry. 2004;13:221-6.

18. Baio J. Prevalence of autism spectrum disorder among children aged 8 years - autism and developmental disabilities monitoring network, 11 sites, United States, 2010. MMWR Surveill Summ. 2014;63:1-21.

19. Fombonne E. Epidemiology of pervasive developmental disorders. Pediatr Res. 2009;65:591-8. 
20. Kang HJ, Kawasawa Yl, Cheng F, Zhu Y, Xu X, Li M, et al. Spatio-temporal transcriptome of the human brain. Nature. 2011;478:483-9.

21. Tottenham N, Tanaka JW, Leon AC, McCarry T, Nurse M, Hare TA, et al. The NimStim set of facial expressions: judgments from untrained research participants. Psychiatry Res. 2009;168:242-9.

22. De Rubeis S, He X, Goldberg AP, Poultney CS, Samocha K, Cicek AE, et al. Synaptic, transcriptional and chromatin genes disrupted in autism. Nature. 2014;515:209-15

23. Iossifov I, O'Roak BJ, Sanders SJ, Ronemus M, Krumm N, Levy D, et al. The contribution of de novo coding mutations to autism spectrum disorder. Nature. 2014:515:216-21.

24. Sanders SJ, He X, Willsey AJ, Ercan-Sencicek AG, Samocha KE, Cicek AE, et al. Insights into autism spectrum disorder genomic architecture and biology from 71 risk loci. Neuron. 2015;87:1215-33.

25. Hoischen A, Krumm N, Eichler EE. Prioritization of neurodevelopmental disease genes by discovery of new mutations. Nat Neurosci. 2014;17:764-72.

26. Ronemus $M$, lossifov I, Levy D, Wigler $M$. The role of de novo mutations in the genetics of autism spectrum disorders. Nat Rev Genet. 2014;15:133-41.

27. Bunik V, Kaehne T, Degtyarev D, Shcherbakova T, Reiser G. Novel isoenzyme of 2-oxoglutarate dehydrogenase is identified in brain, but not in heart. FEBS J. 2008;275:4990-5006.

28. Allison T, Ginter H, McCarthy G, Nobre AC, Puce A, Luby M, et al. Face recognition in human extrastriate cortex. J Neurophysiol. 1994;71:821-5.

29. Kanwisher N, McDermott J, Chun MM. The fusiform face area: a module in human extrastriate cortex specialized for face perception. J Neurosci. 1997 17:4302-11.

30. Pitcher D, Walsh V, Duchaine B. The role of the occipital face area in the cortical face perception network. Exp Brain Res. 2011;209:481-93.

31. Schultz RT, Gauthier I, Klin A, Fulbright RK, Anderson AW, Volkmar F, et al. Abnormal ventral temporal cortical activity during face discrimination among individuals with autism and Asperger syndrome. Arch Gen Psychiatry. 2000;57:331-40

32. Pelphrey KA, Sasson NJ, Reznick JS, Paul G, Goldman BD, Piven J. Visual scanning of faces in autism. J Autism Dev Disord. 2002;32:249-61.

33. Klin A, Jones W, Schultz R, Volkmar F, Cohen D. Visual fixation patterns during viewing of naturalistic social situations as predictors of social competence in individuals with autism. Arch Gen Psychiatry. 2002;59:809-16.

34. Papagiannopoulou EA, Chitty KM, Hermens DF, Hickie IB, Lagopoulos J. A systematic review and meta-analysis of eye-tracking studies in children with autism spectrum disorders. Soc Neurosci. 2014;9:610-32.

35. Campbell DJ, Shic F, Macari S, Chawarska K. Gaze response to dyadic bids at 2 years related to outcomes at 3 years in autism spectrum disorders: a subtyping analysis. J Autism Dev Disord. 2014;44:431-42.

36. Norbury CF, Brock J, Cragg L, Einav S, Griffiths H, Nation K. Eye-movement patterns are associated with communicative competence in autistic spectrum disorders. J Child Psychol Psychiatry. 2009;50:834-42.

37. Lewkowicz DJ, Hansen-Tift AM. Infants deploy selective attention to the mouth of a talking face when learning speech. Proc Natl Acad Sci U S A. 2012;109:1431-6.

38. Norbury CF, Griffiths H, Nation K. Sound before meaning: word learning in autistic disorders. Neuropsychologia. 2010;48:4012-9.

39. Morton J, Johnson MH. CONSPEC and CONLERN: a two-process theory of infant face recognition. Psychol Rev. 1991;98:164-81.

40. Johnson MH. Autism: demise of the innate social orienting hypothesis. Curr Biol. 2014;24:R30-1.

41. Jones W, Klin A. Attention to eyes is present but in decline in 2-6-monthold infants later diagnosed with autism. Nature. 2013;504:427-31.

42. Tarpey PS, Smith R, Pleasance E, Whibley A, Edkins S, Hardy C, et al. A systematic, large-scale resequencing screen of $\mathrm{X}$-chromosome coding exons in mental retardation. Nature. 2009;41:535-43.

43. Rauch A, Wieczorek D, Graf E, Wieland T, Endele S, Schwarzmayr T, et al. Range of genetic mutations associated with severe non-syndromic sporadic intellectual disability: an exome sequencing study. Lancet. 2012;380:1674-82.

44. Hu H, Haas SA, Chelly J, Van Esch H, Raynaud M, de Brouwer AP, et al. Xexome sequencing of 405 unresolved families identifies seven novel intellectual disability genes. Mol Psychiatry. 2016;21:133-48.

45. Kirov G, Rees E, Walters JTR, Escott-Price V, Georgieva L, Richards AL, et al. The penetrance of copy number variations for schizophrenia and developmental delay. Biol Psychiatry. 2014;75:378-85.
46. Mulle JG, Dodd AF, McGrath JA, Wolyniec PS, Mitchell AA, Shetty AC, et al. Microdeletions of 3q29 confer high risk for schizophrenia. Am J Hum Genet. 2010;87:229-36.

47. Allen AS, Berkovic SF, Cossette P, Delanty N, Dlugos D, Eichler EE, et al. De novo mutations in epileptic encephalopathies. Nature. 2013;501:217-21.

48. Xu B, lonita-Laza I, Roos JL, Boone B, Woodrick S, Sun Y, et al. De novo gene mutations highlight patterns of genetic and neural complexity in schizophrenia. Nat Genet. 2012;44:1365-9.

49. Fitzgerald TW, Gerety SS, Jones WD, van Kogelenberg M, King DA, McRae J, et al. Large-scale discovery of novel genetic causes of developmental disorders. Nature. 2015:519:223-8.

50. Ahn K, Gotay N, Andersen TM, Anvari AA, Gochman P, Lee Y, et al. High rate of disease-related copy number variations in childhood onset schizophrenia. Mol Psychiatry. 2014;19:568-72.

51. Lim ET, Raychaudhuri S, Sanders SJ, Stevens C, Sabo A, MacArthur DG, et al. Rare complete knockouts in humans: population distribution and significant role in autism spectrum disorders. Neuron. 2013;77:235-42.

52. Piton A, Gauthier J, Hamdan FF, Lafreniere RG, Yang Y, Henrion E, et al. Systematic resequencing of $X$-chromosome synaptic genes in autism spectrum disorder and schizophrenia. Mol Psychiatry. 2011;16:867-80.

53. Niranjan TS, Skinner C, May M, Turner T, Rose R, Stevenson R, et al. Affected kindred analysis of human $\mathrm{X}$ chromosome exomes to identify novel $\mathrm{X}$ linked intellectual disability genes. PLoS One. 2015;10:e0116454

54. Fromer M, Pocklington AJ, Kavanagh DH, Williams HJ, Dwyer S, Gormley P, et al. De novo mutations in schizophrenia implicate synaptic networks. Nature. 2014:506:179-84.

55. de Ligt J, Willemsen MH, van Bon BW, Kleefstra T, Yntema HG, Kroes T, et al. Diagnostic exome sequencing in persons with severe intellectual disability. N Engl J Med. 2012;367:1921-9.

\section{Submit your next manuscript to BioMed Central and we will help you at every step:}

- We accept pre-submission inquiries

- Our selector tool helps you to find the most relevant journal

- We provide round the clock customer support

- Convenient online submission

- Thorough peer review

- Inclusion in PubMed and all major indexing services

- Maximum visibility for your research

Submit your manuscript at www.biomedcentral.com/submit 